For numbered affiliations see end of article.

Correspondence to: R A Mustafa rmustafa@kumc.edu, Q Hao haoqiukui@gmail.com

Additional material is published online only. To view please visit the journal online

Cite this as: BMJ 2021;373:n1091 http://dx.doi.org/10.1136/bmj.n1091

RAPID RECOMMENDATIONS

\title{
SGLT-2 inhibitors or GLP-1 receptor agonists for adults with type 2 diabetes: a clinical practice guideline
}

\author{
Sheyu Li, 1, 2 Per Olav Vandvik, 3,4 Lyubov Lytvyn, ${ }^{5}$ Gordon H Guyatt, ${ }^{5,6}$ Suetonia C Palmer, ${ }^{7}$ \\ René Rodriguez-Gutierrez, 8, 9, 10 Farid Foroutan, ${ }^{11}$ Thomas Agoritsas, ${ }^{12}$ Reed A C Siemieniuk, 5, 6 \\ Michael Walsh, ${ }^{5,6}$ Lawrie Frere, ${ }^{13}$ David J Tunnicliffe, ${ }^{14,15}$ Evi V Nagler, ${ }^{16}$ Veena Manja, ${ }^{17}$ \\ Bjørn Olav Åsvold, ${ }^{18,19}$ Vivekanand Jha, ${ }^{20,22}$ Mieke Vermandere, ${ }^{23}$ Karim Gariani, ${ }^{12}$ Qian Zhao, ${ }^{24}$ Yan Ren, \\ Emma Jane Cartwright, ${ }^{25}$ Patrick Gee, ${ }^{26}$ Alan Wickes, ${ }^{27}$ Linda Ferns, ${ }^{27}$ Robin Wright, ${ }^{27}$ Ling Li, ${ }^{2}$ \\ Qiukui Hao, ${ }^{5,28}$ Reem A Mustafa ${ }^{5,29}$
}

\section{ABSTRACT \\ CLINICAL QUESTION}

What are the benefits and harms of sodium-glucose cotransporter 2 (SGLT-2) inhibitors and glucagon-like peptide 1 (GLP-1) receptor agonists when added to usual care (lifestyle interventions and/or other diabetes drugs) in adults with type 2 diabetes at different risk for cardiovascular and kidney outcomes?

\section{CURRENT PRACTICE}

Clinical decisions about treatment of type 2 diabetes have been led by glycaemic control for decades. SGLT-2 inhibitors and GLP-1 receptor agonists are traditionally used in people with elevated glucose level after metformin treatment. This has changed through trials demonstrating atherosclerotic cardiovascular disease (CVD) and chronic kidney disease (CKD) benefits independent of medications' glucose-lowering potential.

\section{RECOMMENDATIONS}

The guideline panel issued risk-stratified recommendations concerning the use of SGLT-2 inhibitors or GLP-1 receptor agonists in adults with type 2 diabetes

- Three or fewer cardiovascular risk factors without established CVD or CKD: Weak recommendation against starting SGLT-2 inhibitors or GLP-1 receptor agonists.

- More than three cardiovascular risk factors without established CVD or CKD: Weak recommendation for starting SGLT-2 inhibitors and weak against starting GLP-1 receptor agonists.

- Established CVD or CKD: Weak recommendation for starting SGLT-2 inhibitors and GLP-1 receptor agonists.

- Established CVD and CKD: Strong recommendation for starting SGLT-2 inhibitors and weak recommendation for starting GLP-1 receptor agonists. - For those committed to further reducing their risk for CVD and CKD outcomes: Weak recommendation for starting SGLT-2 inhibitors rather than GLP-1 receptor agonists.

HOW THIS GUIDELINE WAS CREATED

An international panel including patients, clinicians, and methodologists created these recommendations following standards for trustworthy guidelines and using the GRADE approach. The panel applied an individual patient perspective.

\section{THE EVIDENCE}

A linked systematic review and network meta-analysis (764 randomised trials included 421346 participants) of benefits and harms found that SGLT-2 inhibitors and GLP-1 receptor agonists generally reduce overall death, and incidence of myocardial infarctions, and end-stage kidney disease or kidney failure (moderate to high certainty evidence). These medications exert different effects on stroke, hospitalisations for heart failure, and key adverse events in different subgroups. Absolute effects of benefit varied widely based on patients' individual risk (for example, from five fewer deaths in the lowest risk to 48 fewer deaths in the highest risk, for 1000 patients treated over five years). A prognosis review identified 14 eligible risk prediction models, one of which (RECODe) informed most baseline risk estimates in evidence summaries to underpin the risk-stratified recommendations. Concerning patients' values and preferences, the recommendations were supported by evidence from a systematic review of published literature, a patient focus group study, a practical issues summary, and a guideline panel survey.

\section{UNDERSTANDING THE RECOMMENDATION}

We stratified the recommendations by the levels of risk for CVD and CKD and systematically considered the balance of benefits, harms, other considerations, and practical issues for each risk group. The strong recommendation for SGLT-2 inhibitors in patients with CVD and CKD reflects what the panel considered to be a clear benefit. For all other adults with type 2 diabetes, the weak recommendations reflect what the panel considered to be a finer balance between benefits, harms, and burdens of treatment options. Clinicians using the guideline can identify their patient's individual risk for cardiovascular and kidney outcomes using credible risk calculators such as RECODe. Interactive evidence summaries and decision aids may support well informed treatment choices, including shared decision making.

People with type 2 diabetes (a condition with an increasing prevalence globally ${ }^{12}$ ) face an increased risk of cardiovascular disease, kidney disease, and

This BMJ Rapid Recommendation article is one of a series that provides clinicians with trustworthy recommendations for potentially practice changing evidence. BMJ Rapid Recommendations represent a collaborative effort between the MAGIC group (https://magicevidence.org/) and The BMJ. A summary is offered here and the full version including decision aids is on the MAGICapp (https://app.magicapp.org/), for 
other complications. ${ }^{3}$ For decades, management of type 2 diabetes has been led by blood glucose and glycated haemoglobin (HbA1c) treatment targets, 45 but recent high quality randomised controlled trials have challenged this glucocentric paradigm, with outcomes suggesting that intensive glycaemic control may not always correlate with a reduction in macrovascular outcomes and may be associated with harm. ${ }^{67}$

Regulatory agencies are now requiring new diabetes medications to demonsrate benefit on cardiovascular and kidney outcomes to obtain approval. Trials of two newer classes of medication-sodium-glucose cotransporter 2 (SGLT-2) inhibitors and glucagon-like peptide 1 (GLP-1) receptor agonists (see box 1)-suggest that these drugs, when added to existing treatment regimens (usual care), demonstrate benefits on death, myocardial infarctions, and stroke and, more recently, heart failure and kidney outcomes such as progression to end stage kidney disease. ${ }^{8-12}$ However, although systematic reviews show consistent relative risk reductions, the drugs' absolute benefits and harms depend on patients' individual risk profiles. ${ }^{13}$

Box 1: What are sodium-glucose cotransporter 2 (SGLT-2) inhibitors and glucagon-like peptide 1 (GLP-1) receptor agonists?

SGLT-2 inhibitors are a class of oral anti-diabetic drugs, including empagliflozin, canagliflozin, dapagliflozin, and ertugliflozin. They increase the excretion of glucose and sodium in the urine by inhibiting SGLT-2 in the kidney, thus lowering the blood glucose level. They may also slightly lower blood pressure and body weight.

GLP-1 receptor agonists are a class of non-insulin injection anti-diabetic drugs, including exenatide, liraglutide, lixisenatide, albiglutide, dulaglutide, semaglutide, and loxenatide. They mimic the intestinal hormone incretin and bind its receptor, which slows the rate at which food leaves the stomach, controls the appetite, and regulates insulin and glucagon secretion.

This guideline represents a shift from the traditional focus on glycaemic control to a focus on the absolute reduction of cardiovascular and kidney disease outcomes. We provide risk-stratified recommendations about when to add SGLT-2 inhibitors or GLP-1 receptor agonists to existing treatment for adults with type 2 diabetes. The recommendations are based on patients' individual risk of cardiovascular and kidney diseases that determine the anticipated absolute benefits of SGLT-2 inhibitors and GLP-1 receptor agonists. These benefits need to be carefully weighed against potential harms and practical issues resulting from adding these medications to usual care.

The infographic provides an overview of the recommendations, with evidence summaries displaying benefits, harms, and practical issues. Box 2 shows all the evidence linked in this Rapid Recommendation package, with linked systematic reviews on effectiveness, ${ }^{13}$ prognosis, ${ }^{14}$ and patients' values and preferences ${ }^{15}$ underpinning the recommendations.

\section{Box 2: Linked resources for this BMJ Rapid Recommendations cluster}

- Li S, Vandvik PO, Lytvyn L, et al. SGLT-2 inhibitors or GLP-1 receptor agonists for adults with type 2 diabetes: a clinical practice guideline. BMJ 2021;373:n1091 doi:10.1136/bmj.n1091

Summary of the results from the Rapid Recommendation process

- Palmer SC, Tendal B, Mustafa RA, et al. Sodium-glucose transport protein 2 (SGLT-2) inhibitors and glucagon-like peptide-1 (GLP-1) receptor agonists for type 2 diabetes: A systematic review and network meta-analysis of randomised controlled trials. BMJ 2021;372:m4573 doi:10.1136/bmj.m4573

- Rodriguez-Gutierrez R, et al. Values, preferences and burden of treatment for the initiation of GLP-1 receptor agonists and SGLT-2 inhibitors in adult patients with type 2 diabetes: a systematic review. BMJ Open [forthcoming]

- Buchan TA, Malik A, Chan C, et al. Predictive models for cardiovascular and kidney outcomes in patients with type 2 diabetes: systematic review and meta-analyses. Heart 2021; doi:10.1136/heartjnl-2021319243

- MAGICApp. https://app.magicapp.org/\#/guideline/4676

- Expanded version of the results with multilayered recommendations, evidence summaries, and decision aids for use on all devices

\section{Current practice}

Existing guidelines vary in their approach to using newer evidence about macrovascular outcomes or traditional glucocentric approaches to determine guidance about treatment (table 1). ${ }^{23}$ Professional societies within cardiology, nephrology, and diabetes $^{17-22}$ increasingly recommend SGLT-2 inhibitors or GLP-1 receptor agonists in adults at high cardiovascular risks, including those with established cardiovascular disease, heart failure, and chronic kidney disease. ${ }^{17-22}$ Other prominent guideline organisations (such as the National Institute for Health and Care Excellence, NICE) still apply a glucocentric approach and recommend an alternative initial medication for most adults with type 2 diabetes, leaving SGLT-2 inhibitors and GLP-1 receptor agonists as alternative options. ${ }^{16}$ Existing guidelines do not provide clear judgments on how the balance of benefits and harms of these drugs vary across patients with type 2 diabetes and different cardiovascular and/or kidney risk, nor do they report how patients' values and preferences were considered in developing their recommendations.

\section{How these recommendations were created}

\section{Who was involved?}

We recruited an international guideline panel with patient partners (people living with type 2 diabetes with or without complications), general practitioners, general internists, endocrinologists, nephrologists, cardiologists, geriatricians, and methodologists. The panel decided on the scope of this guideline and formulated recommendations. No panel member reported financial conflicts of interest. Intellectual conflicts of interest were minimised and managed (see appendix 1 on bmj.com for details of panel members and their competing interests).

\section{What research did the guideline panel request and review?}

To fully inform their clinical question the panel identified the need for three linked systematic reviews: 1 ) on benefits and harms of starting SGLT-2 inhibitors and GLP-1 receptor agonists to existing therapeutic strategies, including a network meta-analysis and subgroup analyses on key patient characteristics; 2) on prognosis and risk stratification of patients with different risks of cardiovascular and kidney diseases; and 3) on values and preferences of adults with type 2 diabetes regarding SGLT-2 inhibitors and GLP-1 receptor agonists. The panel also requested a focus group study of adults with type 2 diabetes, to better understand the magnitude of benefits, harms, and practical issues that patients consider important in deciding whether to add SGLT-2 inhibitors and GLP-1 receptor agonists, each compared with usual care as well as each other.

What outcomes did the guideline panel consider?

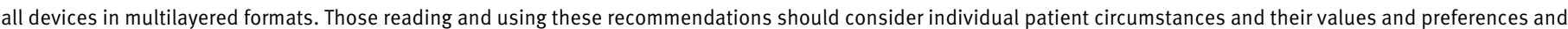

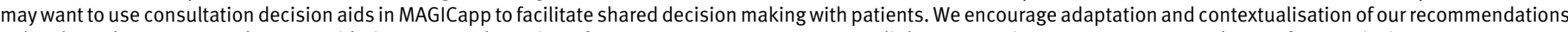

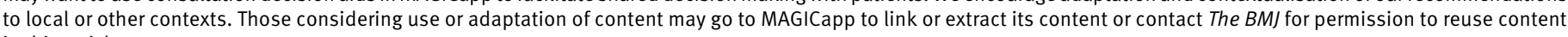
in this article. 
The panel identified 18 patient-important outcomes on benefits and harms to be addressed in the systematic review and network meta-analysis of effectiveness, ${ }^{13}$ including all-cause death, non-fatal myocardial infarction, non-fatal stroke, kidney failure, hospitalisation for heart failure, severe hypoglycaemia, eye disease requiring intervention, health related quality of life, body weight, amputation, neuropathic pain, diabetic ketoacidosis, serious hyperglycaemia, genital infection, Fournier gangrene, severe gastrointestinal events, pancreatic cancer, and pancreatitis. After carefully examining GRADE evidence summaries reporting the absolute effects of starting SGLT-2 inhibitors and GLP-1 receptor agonists in addition to usual care across all these outcomes, the panel ended up focusing on eight key outcomes, of which five concerned benefit (all-cause death, non-fatal myocardial infarction, non-fatal stroke, end stage kidney disease, and hospital admission for heart failure) and three reflected key adverse events for each medication (diabetic ketoacidosis, genital infections, and severe gastrointestinal events).

\section{How did the guideline panel formulate the recommendations?}

The panel followed the $B M$ J Rapid Recommendations procedures for developing trustworthy guidelines ${ }^{39}$ with standards, methods, and processes as detailed in MAGICapp (https://app.magicapp.org). The panel applied the GRADE approach to critically appraise the evidence and create recommendations from a patient perspective. With GRADE, recommendations can be strong or weak, for or against a course of action. $4^{\circ}$ To facilitate the panel's deliberations on the strength and direction of recommendations for each of the five risk groups, we conducted an anonymous panel survey before the teleconferences. For each risk category, we presented the evidence for all benefit and harm outcomes, and then asked the panel about the proportion of participants who would choose either the medication or standard care, as follows: all or almost all (90-100\%) would choose, most (75-90\%) would choose, the majority (51-74\%) would choose, the majority (51-74\%) would decline, most (75-90\%) would decline, all or almost all (90-100\%) would decline. The surveys were not intended to be used akin to votes, but rather to anchor the conversation and facilitate consensus.

During the teleconferences, the panel reviewed the results of the survey, had full discussions aiming to reach consensus, and, when needed, voted to make final recommendations. Recommendations considered the evidence to decision domains including the balance of benefits, harms, and burdens of SGLT-2 inhibitors and GLP-1 receptor agonists, the certainty of the evidence, typical and expected variations in patients' values and preferences, feasibility, acceptability, and equity issues. ${ }^{1441}$ Resource and cost-effectiveness considerations were discussed but were not considered when making recommendations.

As outlined below, the absolute benefits of SGLT-2 inhibitors and GLP-1 receptor agonists depend on a patient's baseline risk-that is, their individual risk for cardiovascular and kidney diseases. ${ }^{14}$ The guideline panel stratified five broad groups of adults with type 2 diabetes based on their baseline risks that clinicians would be able to identify in their practice. The most credible risk prediction model identified in the linked systematic review ${ }^{14}$ informed decisions about baseline risks for cardiovascular and kidney diseases (except hospital admission for heart failure), based on 1) three or fewer cardiovascular risk factors; 2) more than three cardiovascular risk factors; 3) established cardiovascular disease (CVD) without chronic kidney disease (CKD); 4) established CKD without CVD; 5) established CVD and CKD. The control arm of included trials or large observational studies informed decisions about baseline risks for hospital admission for heart failure and harms.

\section{The evidence}

\section{Benefits and harms of SGLT-2 inhibitors and GLP-1 receptor agonists}

The linked systematic review and network meta-analysis (NMA) included 764 randomised controlled trials with 421346 participants with type 2 diabetes. ${ }^{13}$ In brief, the NMA found that SGLT- 2 inhibitors and GLP-1 receptor agonists both reduce all-cause death, cardiovascular death, myocardial infarction, end stage kidney disease and serious hyperglycaemia (all high certainty evidence) as well as potentially lowering body weight (low certainty) with no difference in severe hypoglycaemia (high certainty).

High certainty evidence demonstrated potentially important benefits of SGLT-2 inhibitors over GLP-1 receptor agonists for all-cause death and hospitalisation for heart failure, and of GLP-1 receptor agonists over SGLT-2 inhibitors on non-fatal stroke. Harms also differed, with SGLT-2 inhibitors increasing the risk of diabetic ketoacidosis and genital infection (moderate to high certainty), while GLP-1 receptor agonists may increase the risk of severe gastrointestinal events (low certainty).

None of the subgroup analyses requested by the panel provided credible evidence of different relative effects based on key patient characteristics (such as established versus not established cardiovascular disease).

The relative effects on cardiovascular and renal outcomes were found to be consistent across patients at different risk of such outcomes and for the drug classes, the absolute effects are determined by patients' individual risk profiles (for example, from five fewer deaths in lowest risk to 48 fewer deaths in the highest risk, per 1000 patients treated over five years). The infographic, the linked NMA, ${ }^{13}$ and the MAGICApp interactive decision support tool MATCH-IT display the absolute effects of starting SGLT-2 inhibitors and GLP-1 receptor agonists compared with not starting them (usual care) and against each other in the five risk groups of patients defined for this guideline.

\section{Prognosis and risk prediction for cardiovascular and kidney outcomes}

The prognosis systematic review, including 14 available risk prediction models for adults with type 2 diabetes, identified one model (RECODe) to best predict all-cause death, end stage kidney disease, myocardial infarction, and stroke. ${ }^{142425}$ Appendix 2 provides details about the populations used to develop and validate the RECODe model.

To create GRADE evidence summaries for the five different risk groups, we estimated the baseline risks of these outcomes by simulating typical patients using the RECODe calculator (https://sanjaybasu.shinyapps.io/recode). ${ }^{24}$ Since RECODe reports composite outcomes of myocardial infarction and stroke, we estimated myocardial infarction and stroke by splitting outcomes in a 1:1 ratio. We estimated cardiovascular death to be $2 / 3$ of all-cause death. Since the baseline risk for end stage kidney disease/kidney failure seems to be overestimated for adults with diabetes with no established cardiovascular or kidney disease (the first and second risk groups), we used data from the Study of Diabetes in New Zealand (https://www.nzssd.org.nz/cvd_renal/) to estimate the baseline risk. The panel relied on well performed, large observational studies ${ }^{25}$ and the control arms of included trials to assess the baseline risk for hospital admission for heart failure and harms (diabetic ketoacidosis, genital infection, severe gastrointestinal events).

\section{How do people value benefits and harms?}

The linked systematic review of the patients' values and preferences included 17 studies with 6986 adults with type 2 diabetes. ${ }^{15}$ The review search did not retrieve any published studies regarding patient preferences for SGLT-2 inhibitors and very limited empirical evidence to inform judgments of values and preferences for GLP-1 receptor agonists. People with type 2 diabetes preferred oral medication over injectable treatment, once weekly injections over once daily injections, and simplicity in injection devices. 
The panel therefore convened a focus group with seven patient partners to further elucidate values and preferences of adults with type 2 diabetes. Patient partners were presented with the harms and burdens of starting SGLT-2 inhibitors and GLP-1 receptor agonists, compared with not starting them (usual care). Then the investigators presented potential magnitudes of benefit and sought to determine the threshold at which the benefit outcome would be sufficiently large for participants to accept the harms and burdens of taking additional medications. Of the seven participants, two were willing to accept very small benefits to use either drug $(<5$ in 1000 patients risk reduction in five years), while two would decline either drug even given the largest possible benefit (>30 in 1000 patients risk reduction in five years). Participants weighed avoiding kidney failure similarly to avoiding death, and more highly than avoiding myocardial infarction, stroke, and hospitalisation due to heart failure. Similar to the systematic review of values and preferences, the focus group participants strongly preferred oral medications over injectables.

\section{Understanding the recommendations}

\section{Who do they apply to?}

This clinical practice guideline is aimed at clinicians caring for people with type 2 diabetes and considering adding an SGLT-2 inhibitor or a GLP-1 receptor agonist to existing treatment regardless of the patient's ethnicity, gender, HbA1c levels, comorbidities, or underlying risk of cardiovascular and/or kidney disease.

The discussion regarding starting these drugs is typically to help manage long term diabetes complications and are not meant for short term glucose management.

\section{What is my patient's risk?}

Applying the risk-stratified recommendations requires clinicians to identify their patients' individual risk profiles for cardiovascular and kidney outcomes. It is not difficult to categorise patients with established cardiovascular and/or kidney diseases. In the absence of established disease, clinicians would need to estimate the number of risk factors, including but not limited to age over 60 years old, male, family history of cardiovascular or kidney disease, uncontrolled $\mathrm{HbA1c}(\geq 6.5 \%)$, current smoking, uncontrolled hypertension (>140/90 mm $\mathrm{Hg}$ ), and dyslipidaemias including elevated total cholesterol $(\geq 5.2 \mathrm{mmol} / \mathrm{L})$ and reduced high density lipoprotein (HDL) cholesterol ( $<1 \mathrm{mmol} / \mathrm{L})$. We provide the RECODe tool (available online at https://sanjaybasu.shinyapps.io/recode) for this purpose, given its superior credibility compared with other available tools. ${ }^{14}$ Importantly, this risk prediction model has only been validated in the US population and needs to be used with caution in other populations that may have different cardiovascular and/or kidney disease risk. ${ }^{26}$

\section{$\mathrm{HbA1c}$ and glucose control}

HbA1c has long been used to guide clinical decision making about type 2 diabetes. ${ }^{5}$ However, systematic reviews have revealed minimal benefits in normalisation of HbA1c. ${ }^{523}$ Moreover, the cardiovascular and kidney protection of SGLT-2 inhibitors and GLP-1 receptor agonists are unrelated to their impact on $\mathrm{HbA1c} .{ }^{27^{-30}}$ It is therefore cardiovascular and kidney risk, rather than $\mathrm{HbA1c}$, that constitutes a possible indication for the two medication classes. All of the trials that demonstrated these drugs' benefit were, however, conducted in patients whose $\mathrm{HbA1c}$ values were $>6.5 \%$. Whether those with a lower $\mathrm{HbA1c}$ would achieve the same benefit is uncertain.

How to manage patients with severe hyperglycaemia falls outside the scope of our guideline. We note that patients with very high blood glucose levels (such as $>16.7 \mathrm{mmol} / \mathrm{L}$ or $\mathrm{HbA1c}>9 \%$ ) are at risk of life threatening severe hyperglycaemia associated with volume depletion, severe infection, and possible ketoacidosis. ${ }^{31}$ Such patients need special evaluations and optimised care, which are addressed by other clinical practice guidelines. ${ }^{17-22}$ Clinicians should also consider the risks of other chronic complications such as retinopathy, cataract, neuropathy, and diabetic foot ulcer in those with high HbA1c levels when making treatment decisions.

\section{Safety and harms}

While the systematic review on benefits and harms generally confirmed the two drugs to be safe, some specific considerations apply to the observed adverse events, together with some potential harms raised as concerns by others. ${ }^{13}$

- There may be an increased risk of gastrointestinal events from GLP-1 receptor agonists-including abdominal pain, nausea, vomiting, and diarrhoea-that could be severe and may lead to the withdrawal of the drug. A "start low, go slow" strategy when initiating GLP-1 receptor agonists may reduce the likelihood of patients experiencing these events. The onset of symptoms may dictate a slower up-titration or, depending on patient preferences, discontinuation.

- The increase in genital infections (for example, vaginitis for females and balanitis for males) from SGLT2-inhibitors is important for decision making. A prior genital infection further increases the risk of infection sevenfold in females and 11-fold in males, ${ }^{25}$ so asking patients about prior genital infection is important when considering SGLT-2 inhibitors. One of the most severe forms of genital infection is Fournier's gangrene, which is very rare but can be fatal. It has been associated with SGLT-2 inhibitors in an observational study, ${ }^{32}$ but our network meta-analysis (NMA) did not confirm this association from 41 899 participants in seven trials. ${ }^{13}$

- Despite the NMA confirming a lack of increased diabetic ketoacidosis from SGLT-2 inhibitors, some other reviews with different inclusion criteria raised a concern of diabetic ketoacidosis linked to the use of SGLT-2 inhibitors. ${ }^{33}$ Clinicians should discuss the risk of ketoacidosis associated with certain scenarios, such as limited eating or drinking, severe diarrhoea, gastrointestinal surgery, very low carbohydrate diets, and excess alcohol intake. Unlike with other diabetes medications, it is reported that diabetic ketoacidosis can occur when glucose is within the normal range in people receiving SGLT-2 inhibitors. ${ }^{19}$

- Amputations have also been linked to SGLT-2 inhibitors in an observational study and the CANVAS trial. 93435 However, the NMA did not find an increased risk of amputation in people receiving SGLT-2 inhibitors, ${ }^{13}$ neither did the CREDENCE trial, ${ }^{10}$ which used the same medication as the CANVAS trial. The low certainty evidence and the very low incidence led the panel not to consider this outcome in their recommendation.

- Patients and clinicians may be concerned about the risk of pancreatic cancer and pancreatitis when starting GLP-1 receptor agonists based on previous observational studies. ${ }^{36}$ However, these concerns were not confirmed by the NMA and other published systematic review. ${ }^{13} 37$

- The FDA did not approve the use of SGLT-2 inhibitors in people with estimated glomerular filtration rates (eGFRs) $<30$ $\mathrm{mL} / \mathrm{min} / 1.73 \mathrm{~m}^{2}$, which was not investigated in the eligible trials of the NMA. ${ }^{13}$ GLP-1 receptor agonists may be more appropriate for people with advanced kidney diseases. 


\section{Practical issues}

We encourage clinicians to use shared decision-making with people with type 2 diabetes in choosing SGLT-2 inhibitors and GLP-1 receptor agonists. Table 2 summarises the practical issues related to use of SGLT-2 inhibitors and GLP-1 receptor agonists.

Typically, when adding SGLT-2 inhibitors or GLP-1 receptor agonists, the existing drug regimen would remain unchanged unless there are contraindications or newly added risks such as hypoglycaemia. For example, GLP-1 receptor agonists should not be used with dipeptidyl peptidase 4 (DPP-4) inhibitors; thus, GLP-1 receptor agonists can be added only if the DPP-4 inhibitors are discontinued. ${ }^{16}$ Although neither SGLT-2 inhibitors nor GLP-1 receptor agonists increase the risk of hypoglycaemia, clinicians need to consider the risks of hypoglycaemia due to other ongoing drugs, especially in those at high risk of hypoglycaemia or cardiovascular disease. People receiving insulin, sulphonylureas, or glinides may need to cut $20-50 \%$ of their dose or transfer to a less intensive regimen (taking drugs less often) if their blood glucose levels are close to the target range.

Many patients have an aversion to injectable drugs. The common form of the GLP-1 receptor agonists involves subcutaneous injections daily (such as liraglutide and lixisenatide) or twice daily (such as exenatide); new preparations require only weekly injections (such as albiglutide, dulaglutide, exenatide synthetic, and semaglutide). Oral semaglutide is now approved by the US Food and Drug Administration and Health Canada, but it is not widely accessible in other countries. A compound preparation of GLP-1 receptor agonists and insulin is available and may not require increasing the frequency of injection. It should be noted that our recommendation regarding GLP-1 receptor agonists is mainly based on the once-daily preparation. The systematic review of values and preferences and focus group suggests a strong patient preference for the lowest possible frequency of injection (for example, patients prefer once-weekly GLP-1 receptor agonists over once-daily injections). ${ }^{15}$ Discussing the administration options with patients considering GLP-1 receptor agonists is essential.

\section{Availability and cost}

SGLT-2 inhibitors and GLP-1 receptor agonists have been widely used in the US, Canada, Europe, and China. However, they are unavailable in many other countries due to cost and local health policy. GLP-1 receptor agonists injectable forms require storage and transportation between $2^{\circ} \mathrm{C}$ and $8^{\circ} \mathrm{C}$ before the first use, which limits its availability in remote regions with travel challenges. Even if available, SGLT-2 inhibitors and GLP-1 receptor agonists are expensive in some countries, and not fully covered or not covered under certain conditions by insurance. The cost effectiveness of these drugs was not considered in the current guideline, because of variation in access and models of healthcare reimbursement internationally. Availability and cost considerations are likely to be important for individual patients and healthcare systems aiming to make appropriate use of limited resources.

\section{Limitations}

There are several limitations to this guideline.

- The current evidence supports the decision making based on cardiovascular and kidney outcomes, but not on the absolute risk or risk reduction of other diabetic complications such as hyperglycaemic crisis, severe systematic infection, retinopathy, neuropathy, and diabetic foot ulcer. Clinicians should consider traditional strategies to prevent these complications in patients.
- The baseline risks were estimated using a single risk calculator (RECODe), which was developed using 9635 patients from the Action to Control Cardiovascular Risk in Diabetes (ACCORD) study in North America. ${ }^{24}$ Cardiovascular and kidney risks may vary across ethnicities, racial backgrounds, and countries. ${ }^{38} \mathrm{We}$ thus suggest using other validated risk calculator if available. Nevertheless, our approach facilitates clinical use when a feasible and localised risk calculator is absent.

- Although we used several methods to incorporate patients' values and preferences (patient partners as co-authors on the panel, primary focus group study, a systematic review of values and preferences, panel survey about patient preferences), we realise this was not a representative sample of adults with type 2 diabetes internationally. Although the values and preferences considerations were consistent across methods, there could be important considerations that we missed.

- We introduced an HbA1c-free strategy of decision making, but we have limited evidence, due to trials inclusion, to support it in patients with $\mathrm{HbA1c}$ lower than $6.5 \%$. Caution is advised when applying the evidence to this population.

\section{Uncertainties}

The following remains uncertain for clinicians and patients

- The potential additional benefit from combining SGLT-2 inhibitors and GLP-1 receptor agonists

- The benefits and harms of using SGLT-2 inhibitors in patients with chronic kidney disease and estimated glomerular filtration rate $<30 \mathrm{~mL} / \mathrm{min} / 1.73 \mathrm{~m}^{2}$

- Validated tools assessing the baseline risk of all critical outcomes for ethnically, racially, and geographically diverse groups of patients are needed

- How patients' values and preferences affect decisions about using different diabetes drugs

- The effect of different diabetes drugs on quality of life, and accurate assessment of harm in longitudinal studies.

\section{Updates to this article}

The steering team of the guideline panel will track newly published evidence and judge whether an update of recommendation is needed when the evidence may change practice.

\section{How patients were involved in the creation of this article}

Four patient partners living with type 2 diabetes were included as full panel members of the guideline (two males and two females; two from the US, one from the UK, and one from South Africa). The panel members identified and prioritised patient-important outcomes, important subgroups, and anchored the discussion on patients' values and preferences. Overall patient partners felt that the cardiovascular and kidney benefit outweigh the harm, but that this could vary considerably among individuals, particularly in terms of access and cost, and the desire to limit the number of medications taken.

Seven participants living with type 2 diabetes were included in a focus group study that informed patients' values and preferences considerations (six male, one female; all from Canada). The participants informed the minimum important difference of benefit, relative outcome priorities, and important practical issues.

\section{AUTHOR AFFILIATIONS}

Department of Endocrinology and Metabolism, West China Hospital, Sichuan University, Chengdu, China 
2

Chinese Evidence-based Medicine Center, Cochrane China Center and MAGIC China Center, West China Hospital, Sichuan University, Chengdu, China

University of Oslo, Oslo, Norway

MAGIC Evidence Ecosystem Foundation

5 Department of Health Research Methods, Evidence and Impact, McMaster University, Hamilton, ON, Canada Department of Medicine, McMaster University, Hamilton, ON, Canada

Department of Medicine, University of Otago, Christchurch, New Zealand

8 Plataforma INVEST Medicina UANL - KER Unit (KER Unit México), Subdirección de Investigación, Universidad Autónoma de Nuevo León, Monterrey, 64460, México

Knowledge and Evaluation Research Unit in Endocrinology, Mayo Clinic, Rochester, MN, 55905, USA

10

Endocrinology Division, Department of Internal Medicine, University Hospital “Dr. José E. González,” Universidad Autónoma de Nuevo León, Monterrey, 64460, México

Ted Rogers Center for Heart Research, Canada

12 Service of Endocrinology, Diabetes, Nutrition and Patient Therapeutic Education, Geneva University Hospitals, Geneva, Switzerland

Newbridge Surgery, Bath, UK

14

Sydney School of Public Health, The University of Sydney, Sydney, Australia

15 Centre for Kidney Research, The Children's Hospital at Westmead, Sydney, Australia

Renal Division, Ghent University Hospital, Belgium

University of California Davis, USA

Department of Endocrinology, Clinic of Medicine, St Olavs Hospital, Trondheim University Hospital, Trondheim, Norway

19 K.G. Jebsen Center for Genetic Epidemiology, Department of Public Health and Nursing NTNU, Norwegian University of Science and Technology, Trondheim, Norway

20

The George Institute for Global Health, UNSW, India

School of Public Health, Imperial College, London, UK

Prasanna School of Public Health, Manipal Academy of Higher Education, Manipal, India

23

Department of Public Health and Primary Care, KU Leuven, Leuven, Belgium

24

International Medical Center / Ward of General Practice, West China Hospital, Sichuan University, Chengdu, China

London, UK

Founder \& CEHD, iAdvocate, Inc., Virginia, Patient partner

27

Patient partner

28

The Center of Gerontology and Geriatrics/National Clinical Research Center for Geriatrics, West China Hospital, Sichuan University, Chengdu, China

Department of Internal Medicine, Division of Nephrology and Hypertension, University of Kansas, Kansas City, USA

Competing interests: All authors have completed the BMJRapid Recommendations interests disclosure form, and a detailed description of all disclosures is reported in appendix 1 on bmi.com. As with all $B M$ Rapid Recommendations, the executive team and The BMJ judged that no panel member had any financial conflict of interest. Professional and academic interests are minimised as much as possible, while maintaining necessary expertise on the panel to make fully informed decisions. Three authors of the systematic review were on the guideline panel (Li L, Suetonia P, Farid F).

Funding: This guideline was funded by the 1.3.5 Project for Disciplines of Excellence, West China Hospital, Sichuan University (grant number ZYGD18022).

Transparency: RA Mustafa and Q Hao affirm that the manuscript is an honest, accurate, and transparent account of the recommendation being reported; that no important aspects of the recommendation have been omitted; and that any discrepancies from the recommendation as planned and registered have been explained.

1 Cho NH, Shaw JE, Karuranga S, etal. IDF Diabetes Atlas: Global estimates of diabetes prevalence for 2017 and projections for 2045. Diabetes Res Clin Pract 2018:138:271-81. doi: 10.1016/j.diabres.2018.02.023 pmid: 29496507

2 Bommer C, Sagalova V, Heesemann E, etal. Global economic burden of diabetes in adults: projections from 2015 to 2030. Diabetes Care 2018;41:963-70. doi: 10.2337/dc17-1962 pmid: 29475843

3 Einarson TR, Acs A, Ludwig C, Panton UH. Prevalence of cardiovascular disease in type 2 diabetes: a systematic literature review of scientific evidence from across the world in 2007-2017. Cardiovasc Diabetol 2018;17:83. doi: 10.1186/s12933-018-0728-6 pmid: 29884191

4 Nathan DMDCCT/EDIC Research Group. The diabetes control and complications tria//epidemiology of diabetes interventions and complications study at 30 years: overview. Diabetes Care 2014;37:9-16. doi: 10.2337/dc13-2112 pmid: 24356592

5 Rodriguez-Gutierrez R, Gonzalez-Gonzalez JG, Zuñiga-Hernandez JA, McCoy RG. Benefits and harms of intensive glycemic control in patients with type 2 diabetes. BMJ2019;367:15887. doi: 10.1136/bmj.l5887 pmid: 31690574

6 Gerstein HC, Miller ME, Byington RP, etalAction to Control Cardiovascular Risk in Diabetes Study Group. Effects of intensive glucose lowering in type 2 diabetes. N Engl J Med 2008;358:2545-59. doi: 10.1056/NEJMoa0802743 pmid: 18539917

7 Patel A, MacMahon S, Chalmers J, etalADVANCE Collaborative Group. Intensive blood glucose control and vascular outcomes in patients with type 2 diabetes. NEnglJ Med2008;358:2560-72 doi: 10.1056/NEJMoa0802987 pmid: 18539916

8 Marx N, Rydén L, Brosius F, etal. Proceedings of the Guideline Workshop 2019 - Strategies for the optimization of guideline processes in diabetes, cardiovascular diseases and kidney diseases. Diabetes Res Clin Pract2020;162:108092. doi: 10.1016/j.diabres.2020.108092 pmid: 32109519

9 Neal B, Perkovic V, Mahaffey KW, etalCANVAS Program Collaborative Group. Canagliflozin and cardiovascular and renal events in type 2 diabetes. N Engl J Med 2017;377:644-57. doi: 10.1056/NEJMoa1611925 pmid: 28605608

10 Perkovic V, Jardine MJ, Neal B, etalCREDENCE Trial Investigators. Canagliflozin and renal outcomes in type 2 diabetes and nephropathy. N Engl / Med 2019;380:2295-306. doi: 10.1056/NEJMoa1811744 pmid: 30990260

11 Gerstein HC Colhoun HM, Dagenais GR etalREWIND Investigators. Dulaglutide and cardiovascula outcomes in type 2 diabetes (REWIND): a double-blind, randomised placebo-controlled trial. Lancet 2019;394:121-30. doi: 10.1016/S0140-6736(19)31149-3 pmid: 31189511

12 Gerstein HC, Colhoun HM, Dagenais GR, etalREWIND Investigators. Dulaglutide and renal outcomes in type 2 diabetes: an exploratory analysis of the REWIND randomised, placebo-controlled trial. Lancet 2019;394:131-8. doi: 10.1016/S0140-6736(19)31150-X pmid: 31189509

13 Palmer SC, Tendal B, Mustafa RA, etal. Sodium-glucose cotransporter protein-2 (SGLT-2) inhibitors and glucagon-like peptide-1 (GLP-1) receptor agonists for type 2 diabetes: systematic review and network meta-analysis of randomised controlled trials. BM/2021;372:m4573. doi: 10.1136/bmj.m4573 pmid: 33441402

14 Rodriguez-Gutierrez R, et al. Values, preferences and burden of treatment for the initiation of GLP-1 receptor agonists and SGLT-2 inhibitors in adult patients with type 2 diabetes: a systematic review. BMJ Open [forthcoming].

15 Buchan TA, Malik A, Chan C, etal. Predictive models for cardiovascular and kidney outcomes in patients with type 2 diabetes: systematic review and meta-analyses. Heart 2021; doi: 10.1136/heartinl-2021-319243 pmid: 33833070

16 National Institute for Health and Care Excellence. Type 2 diabetes in adults: management (NICE guideline NG28). 2020. https://www.nice.org.uk/guidance/ng28.

17 American Diabetes Association. 9. Pharmacologic approaches to glycemic treatment: Standards of Medical Care in Diabetes-2021. Diabetes Care 2021:44(Suppl 1):S111-24 doi: 10.2337/dc21-S009 pmid: 33298420

18 Cosentino F, Grant PJ, Aboyans V, etalESC Scientific Document Group. 2019 ESC Guidelines on diabetes, pre-diabetes, and cardiovascular diseases developed in collaboration with the EASD. Eur Heart /2020;41:255-323. doi: 10.1093/eurhearti/ehz486 pmid: 31497854

19 Garber AJ, Handelsman Y, Grunberger G, etal. Consensus statement by the American Association of Clinical Endocrinologists and American College of Endocrinology on the Comprehensive Type 2 Diabetes Management Algorithm - 2020 executive summary. Endocr Pract 2020;26:107-39. doi: 10.4158/CS-2019-0472 pmid: 32022600

20 Das SR, Everett BM, Birtcher KK, etal. 2020 expert consensus decision pathway on novel therapies for cardiovascular risk reduction in patients with type 2 diabetes: a report of the American College of Cardiology Solution Set Oversight Committee. J Am Coll Cardiol 2020;76:1117-45. doi: 10.1016/j.jacc.2020.05.037 pmid: 32771263 
21 Chinese Diabetes Society, Chinese Society of Endocrinology. Expert consensus on glucose-lowering pharmacotherapies in Chinese adults with type 2 diabetes and cardiovascular disease or chronic kidney disease. Chin J Diabetes Mellitus . 2020;12(6):369-81. doi: 10.3760/cma.j.cn

22 Kidney Disease: Improving Global Outcomes (KDIGO) Diabetes Work Group. KDIGO 2020 clinical practice guideline for diabetes management in chronic kidney disease. Kidney Int 2020;98(4S):S1-115. doi: 10.1016/j.kint.2020.06.019 pmid: 32998798

23 Rodríguez-Gutiérrez R, Montori VM. Glycemic control for patients with type 2 diabetes mellitus: our evolving faith in the face of evidence. Circ Cardiovasc Qual Outcomes 2016;9:504-12. doi: 10.1161/CIRCOUTCOMES.116.002901 pmid: 27553599

24 Basu S, Sussman JB, Berkowitz SA, Hayward RA, Yudkin JS. Development and validation of Risk Equations for Complications Of type 2 Diabetes (RECODe) using individual participant data from randomised trials. Lancet Diabetes Endocrinol 2017;5:788-98. doi: 10.1016/S2213-8587(17)30221-8 pmid: 28803840

25 Hirji I, Andersson SW, Guo Z, Hammar N, Gomez-Caminero A. Incidence of genital infection among patients with type 2 diabetes in the UK General Practice Research Database. J Diabetes Complications 2012;26:501-5. doi: 10.1016/j.jdiacomp.2012.06.012 pmid: 22840886

26 Basu S, Sussman JB, Berkowitz SA, etal. Validation of Risk Equations for Complications of Type 2 Diabetes (RECODe) using individual participant data from diverse longitudinal cohorts in the U.S. Diabetes Care 2018;41:586-95. doi: 10.2337/dc17-2002 pmid: 29269511

27 Palmer SC, Mavridis D, Nicolucci A, etal. Comparison of clinical outcomes and adverse events associated with glucose-lowering drugs in patients with type 2 diabetes: a meta-analysis. JAMA 2016;316:313-24. doi: 10.1001/jama.2016.9400 pmid: 27434443

28 Zheng SL, Roddick AJ, Aghar-Jaffar R, etal. Association between use of sodium-glucose cotransporter 2 inhibitors, glucagon-like peptide 1 agonists, and dipeptidyl peptidase 4 inhibitors with all-cause mortality in patients with type 2 diabetes: a systematic review and meta-analysis. JAMA 2018;319:1580-91. doi: 10.1001/jama.2018.3024 pmid: 29677303

29 Zelniker TA, Wiviott SD, Raz I, etal. SGLT2 inhibitors for primary and secondary prevention of cardiovascular and renal outcomes in type 2 diabetes: a systematic review and meta-analysis of cardiovascular outcome trials. Lancet 2019;393:31-9. doi: 10.1016/S0140-6736(18)32590-X pmid: 30424892

30 Zhu J, Yu X, Zheng Y, etal. Association of glucose-lowering medications with cardiovascular outcomes: an umbrella review and evidence map. Lancet Diabetes Endocrinol 2020;8:192-205. doi: 10.1016/S2213-8587(19)30422-X pmid: 32006518

31 Steenkamp DW, Alexanian SM, McDonnell ME. Adult hyperglycemic crisis: a review and perspective. Curr Diab Rep 2013;13:130-7. doi: 10.1007/s11892-012-0342-z pmid: 23115048
32 Bersoff-Matcha SJ, Chamberlain C, Cao C, Kortepeter C, Chong WH. Fournier gangrene associated with sodium-glucose cotransporter-2 inhibitors: a review of spontaneous postmarketing cases. Ann Intern Med 2019;170:764-9. doi: 10.7326/M19-0085 pmid: 31060053

33 Liu J, Li L, Li S, etal. Sodium-glucose co-transporter-2 inhibitors and the risk of diabetic ketoacidosis in patients with type 2 diabetes: A systematic review and meta-analysis of randomized controlled trials. Diabetes Obes Metab 2020;22:1619-27. doi: 10.1111/dom.14075 pmid: 32364674

34 Ueda P. Svanström H, Melbye M, etal. Sodium glucose cotransporter 2 inhibitors and risk of serious adverse events: nationwide register based cohort study. BMJ2018;363:k4365. doi: 10.1136/bmj.k4365 pmid: 30429124

35 Lupsa BC, Inzucchi SE. Use of SGLT2 inhibitors in type 2 diabetes: weighing the risks and benefits Diabetologia 2018;61:2118-25. doi: 10.1007/s00125-018-4663-6 pmid: 30132031

36 Elashoff M, Matveyenko AV, Gier B, Elashoff R, Butler PC. Pancreatitis, pancreatic, and thyroid cancer with glucagon-like peptide-1-based therapies. Gastroenterology 2011;141:150-6. doi: 10.1053/j.gastro.2011.02.018 pmid: 21334333

37 Li L, Shen J, Bala MM, etal. Incretin treatment and risk of pancreatitis in patients with type 2 diabetes mellitus: systematic review and meta-analysis of randomised and non-randomised studies. BMJ2014;348:g2366. doi: 10.1136/bmj.g2366 pmid: 24736555

38 Zhou YL, Zhang YG, Zhang R, etal. Population diversity of cardiovascular outcome trials and real-world patients with diabetes in a Chinese tertiary hospital. Chin Med J (Engl) 2021; doi: 10.1097/CM9.0000000000001407 pmid: 33734138

39 Siemieniuk RA, Agoritsas T, Macdonald H, Guyatt GH, Brandt L, Vandvik PO. Introduction to BMJ Rapid Recommendations. BM/2016;354:i5191. doi: 10.1136/bmj.i5191 pmid: 27680768

40 Guyatt GH, Oxman AD, Vist GE, etalGRADE Working Group. GRADE: an emerging consensus on rating quality of evidence and strength of recommendations. BMJ 2008;336:924-6. doi: 10.1136/bmj.39489.470347.AD pmid: 18436948

41 Zhang Y, Coello PA, Guyatt GH, etal. GRADE guidelines: 20. Assessing the certainty of evidence in the importance of outcomes or values and preferences-inconsistency, imprecision, and othe domains. J Clin Epidemiol 2019;111:83-93. doi: 10.1016/.j.clinepi.2018.05.011 pmid: 29800687

Main infographic: Summary of recommendations and evidence

Appendix 1: List of the panel members and their declarations of interests. Appendix 2: Brief introduction to the RECODe tool 


\section{Visual summary of recommendation}

i⿱⺈ Population whese recommendations are relevant for all adults
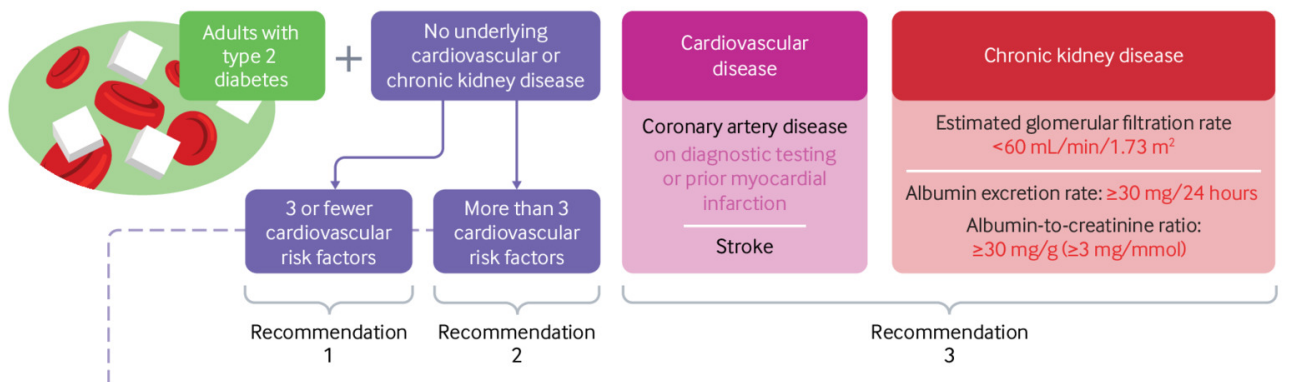

Recommendation

3

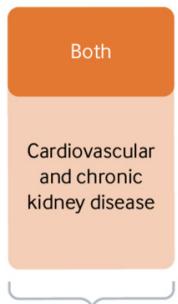

Recommendation 4

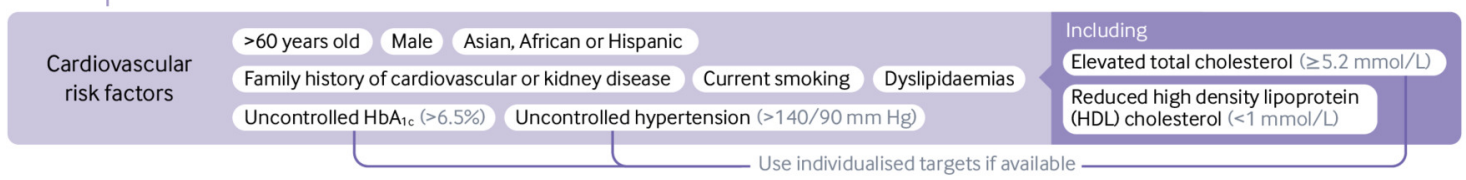

Recommendations SGLT-2 inhibitors

1) Patients with 3 or fewer cardiovascular risk factors

2) Patients with more than 3 cardiovascular risk factors

3) Patients with established cardiovascular or renal disease

4) Patients with established cardiovascular and renal disease

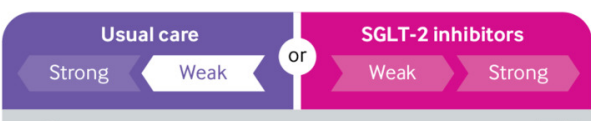

We suggest not using SGLT-2 inhibitors

\section{GLP-1 receptor agonists}

Disclaimer: This infographic is not a validated clinical decision aid. This information is provided without any representations, conditions, or warranties that it is accurate or up to date. BMJ and its licensors assume no responsibility for any aspect of treatment administered with the aid of this information. Any reliance placed on this information is strictly at the user's own risk. For the full dis
see BMJ's terms and conditions: http://www.bmj.com/company/legal-information/

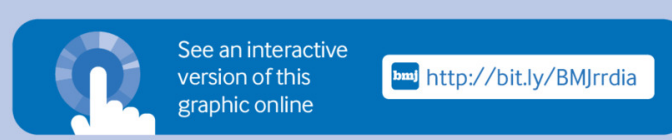


Table 1 | Major guideline recommendations addressing sodium-glucose cotransporter 2 (SGLT-2) inhibitors and glucagon-like peptide 1 (GLP-1) receptor agonists in adults with type 2 diabetes

\begin{tabular}{|c|c|c|}
\hline Guidelines & Recommendations & COR/LOE \\
\hline \multirow[t]{3}{*}{ NICE, $2015^{16}$} & $\begin{array}{l}\text { SGLT-2 inhibitor monotherapy recommended as one of the } \\
\text { options if metformin is contraindicated or intolerant. }\end{array}$ & \multirow[t]{3}{*}{ NA } \\
\hline & $\begin{array}{l}\text { SGLT-2 inhibitors can be used as an option in combination with } \\
\text { metformin and other anti-diabetic drugs. }\end{array}$ & \\
\hline & $\begin{array}{l}\text { GLP-1 receptor agonists suggested as an option if a tripled } \\
\text { anti-diabetic therapy is ineffective, intolerant, or contraindicated } \\
\text { in adults with } \mathrm{BMI} \geq 35 \text { or who may benefit from weight loss. }\end{array}$ & \\
\hline \multirow[t]{3}{*}{ ADA, $2021^{17}$} & $\begin{array}{l}\text { First line therapy is metformin plus comprehensive lifestyle } \\
\text { intervention. }\end{array}$ & \multirow[t]{3}{*}{ NA } \\
\hline & $\begin{array}{l}\text { For patients with established ASCVD or indicators of high ASCVD } \\
\text { risk, either GLP-1 receptor agonists or SGLT-2 inhibitors are } \\
\text { recommended. } \\
\text { For patients with HFrEF, SGLT-2 inhibitors are recommended. } \\
\text { For patients with diabetic kidney diseases and albuminuria, } \\
\text { SGLT-2 inhibitors are recommended, and GLP-1 receptor } \\
\text { agonists are recommended if SGLT-2 inhibitors are intolerant } \\
\text { or contraindicated. For patients with type } 2 \text { diabetes and CKD } \\
\text { (but not diabetic kidney disease or albuminuria), either GLP-1 } \\
\text { receptor agonists or SGLT-2 inhibitors are recommended. }\end{array}$ & \\
\hline & $\begin{array}{l}\text { For patients with and without established ASCVD, but with } \\
\text { HFrEF or CKD, SGLT-2 inhibitors are preferred. }\end{array}$ & \\
\hline ESC, $2019^{18}$ & $\begin{array}{l}\text { In patients with ASCVD or high or very high cardiovascular risks, } \\
\text { SGLT-2 inhibitors or GLP-1 receptor agonists are recommended } \\
\text { to reduce cardiovascular events before inspection of HbA1C } \\
\text { level. }\end{array}$ & I-A \\
\hline AACE, $2020^{19}$ & $\begin{array}{l}\text { Regardless of glucose level, SGLT-2 inhibitors and/or GLP-1 } \\
\text { receptor agonists are recommended in patients with established } \\
\text { or at high risk of ASCVD or CKD. }\end{array}$ & NA \\
\hline ACC, $2020^{20}$ & $\begin{array}{l}\text { SGLT-2 inhibitors or GLP-1 receptor agonists recommended in } \\
\text { adults with type } 2 \text { diabetes and one of ASCVD, heart failure, } \\
\text { or diabetic kidney disease, or at high risk of ASCVD. }\end{array}$ & NA \\
\hline \multirow[t]{2}{*}{ CDS/CSE, $2020^{21}$} & $\begin{array}{l}\text { All adults with type } 2 \text { diabetes need metformin unless } \\
\text { contradicted or not tolerated. }\end{array}$ & $\begin{array}{l}\text { COR: Ila } \\
\text { LOE B }\end{array}$ \\
\hline & $\begin{array}{l}\text { Add GLP-1 receptor agonists or SGLT-2 inhibitors to current } \\
\text { regimen regardless of blood glucose level if patients have } \\
\text { established ASCVD or at high risk of ASCVD and add SGLT-2 } \\
\text { inhibitors to those with heart failure, and SGLT-2 inhibitors to } \\
\text { those with CKD (and GLP-1 receptor agonists if SGLT- } 2 \text { inhibitors } \\
\text { contraindicated). }\end{array}$ & $\begin{array}{l}\text { COR: I } \\
\text { LOE A }\end{array}$ \\
\hline \multirow[t]{3}{*}{ KDIGO, 202022 } & $\begin{array}{l}\text { Metformin recommended in patients with type } 2 \text { diabetes, CKD, } \\
\text { and eGFR } \geq 30 \mathrm{ml} / \mathrm{min} / 1.73 \mathrm{~m} 2 \text {. }\end{array}$ & $1-B$ \\
\hline & $\begin{array}{l}\text { SGLT-2 inhibitors recommended in patients with type } 2 \\
\text { diabetes, CKD, and eGFR } \geq 30 \mathrm{ml} / \mathrm{min} / 1.73 \mathrm{~m} 2 \text {. }\end{array}$ & $1-A$ \\
\hline & $\begin{array}{l}\text { Long-acting GLP-1 receptor agonists recommended in patients } \\
\text { with type } 2 \text { diabetes who have not achieved their individualised } \\
\text { glycaemic target with metformin and SGLT-2 inhibitors. }\end{array}$ & $1-B$ \\
\hline
\end{tabular}

$\mathrm{COR}=$ Class of Recommendation; LOE = Level of Evidence; NICE = National Institute for Health and Care Excellence; ADA/EASD = American Diabetes Association/European Association for the Study of

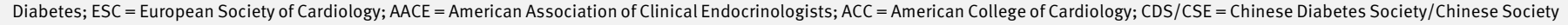
of Endocrinology; KDIGO = Kidney Disease: Improving Global Outcomes.

ASCVD = atherosclerotic cardiovascular disease; $\mathrm{HFrEF}=$ heart failure with reduced ejection fraction. $\mathrm{CKD}=$ chronic kidney disease; eGFR $=$ estimated glomerular filtration rate. 
Table 2 | Practical issues about use of sodium-glucose cotransporter 2 (SGLT-2) inhibitors and glucagon-like peptide 1 (GLP-1) receptor agonists in adults with type 2 diabetes

\begin{tabular}{|c|c|c|}
\hline SGLT-2 inhibitors & GLP-1 receptor agonists & Usual care* \\
\hline \multicolumn{3}{|l|}{ Medication routine } \\
\hline $\begin{array}{l}\text { Tablets swallowed once daily at the same time; some patients } \\
\text { need to take them in the morning. } \\
\text { Combined formulas of SGLT-2 inhibitors and metformin are } \\
\text { available }\end{array}$ & $\begin{array}{l}\text { Injection once or twice daily or once weekly. } \\
\text { Not yet widely available as tablets. } \\
\text { Combined formulas of GLP-1 receptor agonists and insulin are available as a single injection. }\end{array}$ & $\begin{array}{l}\text { Most anti-diabetic drugs are } \\
\text { tablets except insulin, an } \\
\text { injection. } \\
\text { Insulin use may be short term, } \\
\text { such as during an acute illness }\end{array}$ \\
\hline \multicolumn{3}{|c|}{ Should not be taken while a person is sick, especially if there is vomiting, diarrhoea, or the person isn't eating and drinking very much. } \\
\hline \multicolumn{3}{|l|}{ Test and visit } \\
\hline \multicolumn{3}{|c|}{ Regular blood samples, and more frequently after starting a new drug. We only consider adding these drugs when the patient has HbA1c >6.5\% or above their individualised target.. } \\
\hline $\begin{array}{l}\text { We suggest a closer monitoring of blood pressure (within } 1 \\
\text { month or at any time a hypovolaemic dizziness is suspected) } \\
\text { and kidney function (within } 3 \text { months) after newly starting } \\
\text { SGLT-2 inhibitors. }\end{array}$ & & $\begin{array}{l}\text { Self monitoring of blood } \\
\text { glucose for people using } \\
\text { insulin, sulfonylureas, or } \\
\text { meglitinides. }\end{array}$ \\
\hline Recovery and adaptation & & \\
\hline
\end{tabular}

Most people need to take diabetes drugs for the rest of their lives.

\section{Adverse effects, interactions, and antidote}

SGLT-2 inhibitors and GLP-1 receptor agonists do not increase the risk of hypoglycaemia when taken alone, but they do increase the risk when used in combination with some drugs such as insulin, sulfonylureas or glinides

Mild to moderate genital infections can occur. Dose-dependent gastrointestinal adverse reactions, including nausea, vomiting, diarrhoea, Low blood pressure, dizziness, dehydration, etc, especially abdominal pain, indigestion, decreased appetite. These effects are usually most pronounced in among people $>65$ years old or when combined with diuretics. All patients should monitor for signs of diabetic ketoacidosis (even if blood glucose is within the normal range) and seek hospital attention immediately if it occurs.

the first few weeks after starting drug and should be put to special caution in. patients with inflammatory bowel diseases or diabetic gastroparesis. Acute pancreatitis is a rare but serious adverse effect. Should not be used in combination with DPP-4 inhibitors.

\section{Physical wellbeing}

Both drugs have a weight loss effect.

\section{Emotional wellbeing}

Emotional stress can occur from starting or adding new oral or injection drug

Pregnancy and nursing

\section{Both drugs should be avoided during pregnancy and nursing, or in women who may become pregnant.}

Costs and access

Costs vary between specific agents and depend on health insurance and policy. GLP-1 receptor agonists are usually 2-3 times more expensive than SGLT-2 Metformin and sulfonylureas inhibitors. easy to access.

\section{Food and drink}

Keep dietary control in the therapeutic regimen as a general management for type 2 diabetes.

Drinking more water may be

helpful to prevent thirst and dehydration.

\section{Storage and transportation}

before use

No specific considerations

GLP-1 receptor agonists and insulin should be stored and transported at $2-8^{\circ} \mathrm{C}$ before first use.

\section{Exercise and activities}

Keep proper exercise and activities as a general management for type 2 diabetes.

\section{Travel time and driving}

Patients at risk for undetected symptomatic hypoglycaemia need to be aware of the risk of driving. Patients using GLP-1 receptor agonists and insulin may need a special package to bring their drugs and their injection equipment when travelling. The drugs should be stored below $30^{\circ} \mathrm{C}\left(25^{\circ} \mathrm{C}\right.$ for exenatide and benaglutide), avoiding sunshine and freezing after first use. 
Table 2 | Practical issues about use of sodium-glucose cotransporter 2 (SGLT-2) inhibitors and glucagon-like peptide 1 (GLP-1) receptor agonists in adults with type 2 diabetes (Continued)

SGLT-2 inhibitors
FDA label suggests empagliflozin should not be used in patients with eGFR $<45 \mathrm{~mL} / \mathrm{min} / 1.73$

$\mathrm{m} 2$, and canagliflozin, ertugliflozin, and dapagliflozin should not be used in patients with eGFR

$<30 \mathrm{~mL} / \mathrm{min} / 1.73 \mathrm{~m} 2$. All SGLT-2 inhibitors are contraindicated in patients with kidney failure or dialysis.
GLP-1 receptor agonists

Usual care*

DPP-4 = Dipeptidyl peptidase 4; FDA = US Food and Drug Administration; eGFR = estimated glomerular filtration rate.

* Usual care may include some combination of lifestyle intervention, metformin, thiazolidinediones, dipeptidyl peptidase 4 inhibitors, sulfonylureas, meglitinides, $\alpha$-glucosidase inhibitors, and insulin) 